some $C>0$ so that (1) is satisfied by $\gamma=1 / \alpha$. Similarly, if $N(t) \sim t^{\beta} S(t)^{ \pm 1}$, where $\beta>0$ and $S(t)$ is, as in the reference cited, a "slow" function, then (1) holds for every $\gamma<\beta$. If $1 / a_{n}$ is the $n$th prime number, then (1) holds for every $\gamma<1$ since $K(t)$ $>C t^{\gamma}$ in virtue of the elementary inequalities of Tchebycheff. Thus it is not necessary to use the prime number theorem $\beta=1$, $S(t)^{ \pm 1}=1 / \log t$, applied in the reference cited. Correspondingly, the present method enables us to prove (1) also for sequences for which $N(t)$ is not $\sim t^{\beta} S(t)^{ \pm 1}$.

The Johns Hopkins University

\title{
PROOF OF THREE PROPOSITIONS OF PALEY
}

BY M. FEKETE

1. Introduction. In a letter to Fejér,* Paley stated three interesting propositions concerning Fourier series of bounded and continuous functions whose Fourier coefficients satisfy the conditions $n a_{n}, n b_{n} \geqq-K, K \geqq 0$. The letter of Paley contains only a very brief sketch of the proof. After knowing Paley's results the author succeeded in developing complete proofs with various improvements of the estimates, and even in extending them to a wider class of Fourier series of almost periodic functions. These extensions will be treated elsewhere. In the present note we prove the following three theorems.

It will be assumed throughout that $f(x)$ is real-valued and periodic, of period $2 \pi$, and Lebesgue integrable over $(-\pi, \pi)$. Let

$$
f(x) \sim a_{0}+\sum_{n=1}^{\infty}\left(a_{n} \cos n x+b_{n} \sin n x\right)
$$

be its Fourier series expansion and let

$$
s_{n}=s_{n}(x)=a_{0}+\sum_{\nu=1}^{n}\left(a_{\nu} \cos \nu x+b_{\nu} \sin \nu x\right)
$$

* This letter is reproduced in a note by Fejér, On a theorem of Paley, this Bulletin, vol. 40 (1934), pp. 469-475, especially pp. 474-475. It was communicated to the author by Professor Fejér in September, 1933. 
be its partial sums. We also assume that the Fourier coefficients $a_{n}, b_{n}$ satisfy the inequalities

$$
n a_{n} \geqq-K, \quad n b_{n} \geqq-K,
$$

where $K$ is a constant that is not negative.

Theorem A. If $f(x)$ is bounded and

$$
\sup _{x}|f(x)|=L \text {, }
$$

then

$$
\left|s_{n}(x)\right| \leqq 5 L+6 K \text {. }
$$

THEOREM B. Under the same conditions we have

$$
\left|s_{n}(x)\right| \leqq 5 L+8(K L)^{1 / 2} .
$$

Theorem C. If $f(x)$ is continuous and conditions (3) are satisfied, then its Fourier series (1) converges to $f(x)$, uniformly for all $x$.*

2. Proof of Theorem A. The basis of the proof is the classical Fejér inequality, $\left|\sigma_{n}(x)\right| \leqq L$, for the $n$th arithmetic means of the sequence $\left\{s_{n}(x)\right\}$, which being written explicitly reduces to

$$
\left|a_{0}+\sum_{\nu=1}^{n}\left(1-\frac{\nu}{n}\right)\left(a_{\nu} \cos \nu x+b_{\nu} \sin \nu x\right)\right| \leqq L, \quad(n \geqq 1) .
$$

Using the same idea as in the note of Fejér referred to above, we apply (7) to the Fourier series of $2 \phi(x) \cos n x$ and $2 \psi(x) \sin n x$ where

$$
\begin{aligned}
& \phi(x)=\frac{1}{2}\{f(x)+f(-x)\} \sim a_{0}+\sum_{\nu=1}^{\infty} a_{\nu} \cos \nu x \\
& \psi(x)=\frac{1}{2}\{f(x)-f(-x)\} \sim \sum_{\nu=1}^{\infty} b_{\nu} \sin \nu x .
\end{aligned}
$$

* Theorems A and C incorporate the two theorems in Paley's letter, while Theorem B corresponds to his lemma. Theorems A and C were discovered independently, and proved by a different method by Szász, Zur Konvergenztheorie der Fourierschen Reihen, Acta Mathematica, vol. 61 (1933), pp. 185201. Our estimate (5) is sharper than that of Szász, and there is no doubt that it is capable of further improvement. The interest of Theorems A and B lies in the fact that they furnish universal estimates for partial sums $s_{n}(x)$ of all functions of the class under consideration, in terms merely of $L$ and $K$. 
For every $n \geqq 1$ we have

(10) $2 \phi(x) \cos n x \sim \sum_{\nu=0}^{\infty} a_{\nu} \cos (\nu-n) x+\sum_{\nu=0}^{\infty} a_{\nu} \cos (\nu+n) x$,

(11) $2 \psi(x) \sin n x \sim \sum_{\nu=1}^{\infty} b_{\nu} \cos (\nu-n) x-\sum_{\nu=1}^{\infty} b_{\nu} \cos (\nu+n) x$.

On applying (7) we derive

$$
\begin{aligned}
& \mid \sum_{\nu=0}^{n}\left(1-\frac{n-\nu}{n}\right) a_{\nu} \cos (n-\nu) x \\
& \quad+\sum_{\nu=n+1}^{2 n}\left(1-\frac{\nu-n}{n}\right) a_{\nu} \cos (\nu-n) x \mid \leqq 2 L, \\
& \mid \sum_{\nu=1}^{n}\left(1-\frac{n-\nu}{n}\right) b_{\nu} \cos (n-\nu) x \\
& \quad+\sum_{\nu=n+1}^{2 n}\left(1-\frac{\nu-n}{n}\right) b_{\nu} \cos (\nu-n) x \mid \leqq 2 L,
\end{aligned}
$$

whence, for $x=0$,

$$
\begin{aligned}
& \left|\sum_{\nu=1}^{n} \nu a_{\nu}+\sum_{\nu=n+1}^{2 n}(2 n-\nu) a_{\nu}\right| \leqq 2 n L, \\
& \left|\sum_{\nu=1}^{n} \nu b_{\nu}+\sum_{\nu=n+1}^{2 n}(2 n-\nu) b_{\nu}\right| \leqq 2 n L .
\end{aligned}
$$

Formula (14) being combined with (3), yields

$$
0 \leqq \sum_{\nu=1}^{n}\left(\nu a_{\nu}+K\right)+\sum_{\nu=n+1}^{2 n} \frac{2 n-\nu}{\nu}\left(\nu a_{\nu}+K\right) \leqq 2 n(L+K) .
$$

Now, again by (3),

$$
\begin{aligned}
\left|\sum_{\nu=1}^{n} \nu a_{\nu} \cos \nu x\right| & \leqq \sum_{\nu=1}^{n}\left(\nu a_{\nu}+K\right)|\cos \nu x|+K \sum_{\nu=1}^{n}|\cos \nu x| \\
& \leqq(2 L+3 K) n .
\end{aligned}
$$

In similar fashion we derive

$$
\left|\sum_{\nu=1}^{n} \nu b_{\nu} \sin \nu x\right| \leqq(2 L+3 K) n
$$


On combining (7), (16), and (17), we immediately get (5).

3. Proof of Theorem B. We start with the identity indicated by Paley,

$$
s_{n}(x)=\frac{n \sigma_{n}(x)-m \sigma_{m}(x)}{n-m}+R(x), \quad(1 \leqq m<n),
$$

where

(19) $\sigma_{n}(x)=a_{0}+\sum_{\nu=1}^{n}\left(1-\frac{\nu}{n}\right)\left(a_{\nu} \cos \nu x+b_{\nu} \sin \nu x\right)$,

(20) $R(x)=(n-m)^{-1} \sum_{\nu=m+1}^{n}(\nu-m)\left(a_{\nu} \cos \nu x+b_{\nu} \sin \nu x\right)$.

By (7)

$$
\frac{\left|n \sigma_{n}(x)-m \sigma_{m}(x)\right|}{n-m} \leqq \frac{(n+m) L}{n-m}
$$

On applying $(7)$ to the $(n-m)$ th arithmetic means of the series (10) and (11) we have

$$
\begin{aligned}
& \mid \sum_{0 \leqq n-\nu \leqq n-m}\left(1-\frac{n-\nu}{n-m}\right) a_{\nu} \cos (n-\nu) x \\
& \quad+\sum_{0<\nu-n \leqq n-m}\left(1-\frac{\nu-n}{n-m}\right) a_{\nu} \cos (\nu-n) x \mid \leqq 2 L, \\
& \mid \sum_{0 \leqq n-\nu \leqq n-m}\left(1-\frac{n-\nu}{n-m}\right) b_{\nu} \cos (n-\nu) x \\
& \quad+\sum_{0 \leqq \nu-n \leqq n-m}\left(1-\frac{\nu-n}{n-m}\right) b_{\nu} \cos (\nu-n) x \mid \leqq 2 L,
\end{aligned}
$$

whence, for $x=0$,

$$
\begin{aligned}
& \left|\sum_{\nu=m+1}^{n}(\nu-m) a_{\nu}+\sum_{\nu=n+1}^{2 n-m}(2 n-m-\nu) a_{\nu}\right| \leqq 2 L(n-m), \\
& \left|\sum_{\nu=m+1}^{n}(\nu-m) b_{\nu}+\sum_{\nu=n+1}^{2 n-m}(2 n-m-\nu) b_{\nu}\right| \leqq 2 L(n-m) .
\end{aligned}
$$

Formula (24), in view of (3), yields 


$$
\begin{aligned}
0 \leqq & \sum_{\nu=m+1}^{n}(\nu-m)\left(a_{\nu}+K / \nu\right) \leqq \sum_{\nu=m+1}^{n}(\nu-m)\left(a_{\nu}+K / \nu\right) \\
& +\sum_{\nu=n+1}^{2 n-m}(2 n-m-\nu)\left(a_{\nu}+K / \nu\right) \leqq(n-m)\left(2 L+K \sum_{\nu=m+1}^{2 n-m} 1 / \nu\right) .
\end{aligned}
$$

Thus we have

$$
\left|\sum_{\nu=m+1}^{n}(\nu-m) a_{\nu} \cos \nu x\right| \leqq\left|\sum_{\nu=m+1}^{n}(\nu-m)\left(a_{\nu}+K / \nu\right) \cos \nu x\right|
$$

$$
\begin{array}{r}
+\left|\sum_{\nu=m+1}^{n}(\nu-m)(K / \nu) \cos \nu x\right| \\
\leqq \sum_{\nu=m+1}^{n}(\nu-m)\left(a_{\nu}+K / \nu\right)+K \sum_{\nu=m+1}^{n}(\nu-m) / \nu \\
\leqq(n-m)\left(2 L+K \sum_{\nu=m+1}^{2 n-m} 1 / \nu+K \sum_{\nu=m+1}^{n} 1 / \nu\right)
\end{array}
$$

and, similarly,

$$
\begin{aligned}
\mid \sum_{\nu=m+1}^{n}(\nu & -m) b_{\nu} \sin \nu x \mid \\
& \leqq(n-m)\left(2 L+K \sum_{\nu=m+1}^{2 n-m} 1 / \nu+K \sum_{\nu=m+1}^{n} 1 / \nu\right) .
\end{aligned}
$$

Taking into account the well known inequality

$$
\sum_{\nu=\alpha+1}^{\beta} 1 / \nu<\log \frac{\beta}{\alpha}, \quad(1 \leqq \alpha<\beta)
$$

we obtain an estimate for $R(x)$,

$$
|R(x)| \leqq 4 L+2 K \log \left(n(2 n-m) m^{-2}\right), \quad(1 \leqq m<n) .
$$

From (18), (21), (28) it results that, for $1 \leqq m<n$,

$$
\left|s_{n}(x)\right| \leqq L\left(4+\frac{n+m}{n-m}\right)+2 K \log \frac{n(2 n-m)}{m^{2}} .
$$

We propose to show that (29) and (5) imply, for an arbitrary $\delta>0$,

(30) $\left|s_{n}(x)\right| \leqq 5 L+4(L / \delta+K \delta)$. 
If this is shown to be true, then (6) of Theorem B follows at once, since always.

$$
(K L)^{1 / 2} \leqq \frac{1}{2}(L / \delta+K \delta)
$$

with the equality sign (only) in case $\delta=(L / K)^{1 / 2}$ and $K>0$.

To derive (30) from (29), we observe that, unless

$$
n<\frac{1}{2}(2+\delta)\left(1+e^{\delta}\right)\left(e^{\delta}-1-\delta\right)^{-1},
$$

the interval $\left(2 n\left(1+e^{\delta}\right)^{-1}, 2 n(2+\delta)^{-1}\right)$ contains at least one integer $m$. For such an $m$ we have

$$
\begin{aligned}
& 1+\frac{\delta}{2}<\frac{n}{m}<\frac{1}{2}\left(1+e^{\delta}\right)<e^{\delta}, \\
& \frac{n+m}{n-m}=1+2(n / m-1)^{-1}<1+\frac{2}{\delta / 2}=1+\frac{4}{\delta}, \\
& \log \left(n(2 n-m) m^{-2}\right)=\log \left(\frac{n}{m}\right)+\log \left(\frac{2 n}{m}-1\right) \\
& \text { whence, by (29), } \\
& <\log e^{\delta}+\log e^{\delta}=2 \delta, \\
& \left|s_{n}(x)\right| \leqq 5 L+4(L / \delta+K \delta) .
\end{aligned}
$$

Suppose now that (31) holds; (30) follows immediately from (5) if $\delta \geqq 3 / 2$. Finally, if $0<\delta<3 / 2$, then, by the Cauchy-Schwarz inequality and Parseval's theorem,

$$
\begin{aligned}
\left|s_{n}(x)\right| & \leqq\left|a_{0}\right|+\sum_{\nu=1}^{n}\left|a_{\nu} \cos \nu x+b_{\nu} \sin \nu x\right| \\
& \leqq\left|a_{0}\right|+\left(n \sum_{\nu=1}^{n}\left(a_{\nu}^{2}+b_{\nu}^{2}\right)\right)^{1 / 2} \leqq\left(1+(2 n)^{1 / 2}\right) L \\
& \leqq\left\{1+(2+\delta)^{1 / 2}(1+\delta)^{1 / 2}\left(e^{\delta}-1-\delta\right)^{-1 / 2}\right\} L .
\end{aligned}
$$

But, if $0<\delta<3 / 2$, we have

$$
\begin{aligned}
e^{\delta} & <3+4 \delta \\
16(1+\delta)^{2} & >4(1+\delta)\left(1+e^{\delta}\right)>2 \delta^{2}(1+\delta)\left(1+e^{\delta}\right)\left(e^{\delta}-1-\delta\right)^{-i} \\
& >\delta^{2}(2+\delta)\left(1+e^{\delta}\right)\left(e^{\delta}-1-\delta\right)^{-1} \\
1+ & (2+\delta)^{1 / 2}\left(1+e^{\delta}\right)^{1 / 2}\left(e^{\delta}-1-\delta\right)^{-1 / 2}<5+\frac{4}{\delta}
\end{aligned}
$$


and again (30) follows. Thus (30) holds for all $\delta>0$ and the proof of Theorem B is now complete*.

4. Proof of Theorem $\mathrm{C}$. Theorem $\mathrm{C}$ is now easily derived from Theorem B. Since $f(x)$ is uniformly continuous we have by Fejér's theorem,

$$
\max _{x}\left|f(x)-\sigma_{n}(x)\right|=M_{n} \rightarrow 0 \text { as } n \rightarrow \infty .
$$

The Fourier coefficients of the function $f(x)-\sigma_{n}(x)$ are given by

$$
\begin{array}{llrl}
\alpha_{\nu}^{(n)}=\frac{\nu a_{\nu}}{n}, & \beta_{\nu}^{(n)}=\frac{\nu b_{\nu}}{n}, & (1 \leqq \nu \leqq n-1), \\
\alpha_{\nu}^{(n)}=a_{\nu}, & \beta_{\nu}^{(n)}=b_{\nu}, & (\nu \geqq n) .
\end{array}
$$

Conditions (3) obviously imply analogous conditions

$$
\nu \alpha_{\nu}^{(n)} \geqq-K, \quad \nu \beta_{\nu}^{(n)} \geqq-K .
$$

On applying Theorem B to the Fourier series of $f(x)-\sigma_{n}(x)$ we have

$$
\begin{aligned}
\left|s_{n}(x)-\sigma_{n}(x)\right| & =\left|\sum_{\nu=1}^{n}\left(\frac{\nu}{n}\right)\left(a_{\nu} \cos \nu x+b_{\nu} \sin \nu x\right)\right| \\
& \leqq 5 M_{n}+8\left(K M_{n}\right)^{1 / 2}
\end{aligned}
$$

and finally,

$$
\begin{aligned}
\left|f(x)-s_{n}(x)\right| & \leqq\left|f(x)-\sigma_{n}(x)\right|+\left|\sigma_{n}(x)-s_{n}(x)\right| \\
& \leqq 6 M_{n}+8\left(K M_{n}\right)^{1 / 2}
\end{aligned}
$$

This proves Theorem $\mathrm{C}$ and, at the same time, gives information concerning the rapidity with which $s_{n}(x)$ tends to its limiting function $f(x)$.

The Einstein Institute of Mathematics, HEBREW UNIVERSity, JERUSALEM

* Formula (30) is of the type given by Paley, $\left|s_{n}(x)\right| \leqq K \delta+L M_{\delta}$ (loc. cit., p. 474), but has the advantage of giving a simple expression for $M_{\delta}$. 